\title{
Repetitive sequence analysis and karyotyping reveals centromere-associated DNA sequences in radish (Raphanus sativus L.)
}

Qunyan He ${ }^{1,2+}$, Zexi Cai ${ }^{2+}$, Tianhua Hu${ }^{1}$, Huijun Liư ${ }^{2}$ Chonglai Bao ${ }^{1}$, Weihai Mao ${ }^{1 *}$ and Weiwei $\mathrm{Jin}^{2^{*}}$

\begin{abstract}
Background: Radish (Raphanus sativus L., $2 n=2 x=18$ ) is a major root vegetable crop especially in eastern Asia. Radish root contains various nutritions which play an important role in strengthening immunity. Repetitive elements are primary components of the genomic sequence and the most important factors in genome size variations in higher eukaryotes. To date, studies about repetitive elements of radish are still limited. To better understand genome structure of radish, we undertook a study to evaluate the proportion of repetitive elements and their distribution in radish.
\end{abstract}

Results: We conducted genome-wide characterization of repetitive elements in radish with low coverage genome sequencing followed by similarity-based cluster analysis. Results showed that about 31\% of the genome was composed of repetitive sequences. Satellite repeats were the most dominating elements of the genome. The distribution pattern of three satellite repeat sequences (CL1, CL25, and CL43) on radish chromosomes was characterized using fluorescence in situ hybridization (FISH). CL1 was predominantly located at the centromeric region of all chromosomes, CL25 located at the subtelomeric region, and CL43 was a telomeric satellite. FISH signals of two satellite repeats, CL1 and CL25, together with $5 \mathrm{~S}$ rDNA and 45S rDNA, provide useful cytogenetic markers to identify each individual somatic metaphase chromosome. The centromere-specific histone H3 (CENH3) has been used as a marker to identify centromere DNA sequences. One putative CENH3 (RSCENH3) was characterized and cloned from radish. Its deduced amino acid sequence shares high similarities to those of the CENH3s in Brassica species. An antibody against $B$. rapa $\mathrm{CENH3}$, specifically stained radish centromeres. Immunostaining and chromatin immunoprecipitation (ChIP) tests with anti-BrCENH3 antibody demonstrated that both the centromere-specific retrotransposon (CR-Radish) and satellite repeat (CL1) are directly associated with RsCENH3 in radish.

Conclusions: Proportions of repetitive elements in radish were estimated and satellite repeats were the most dominating elements. Fine karyotyping analysis was established which allow us to easily identify each individual somatic metaphase chromosome. Immunofluorescence- and ChIP-based assays demonstrated the functional significance of satellite and centromere-specific retrotransposon at centromeres. Our study provides a valuable basis for future genomic studies in radish.

Keywords: Radish, Repetitive DNA, Satellite, Karyotyping, CENH3, Centromere

\footnotetext{
*Correspondence: maowh@126.com; weiweijin@cau.edu.cn

${ }^{\dagger}$ Equal contributors

'Institute of Vegetables, Zhejiang Academy of Agricultural Sciences,

Hangzhou 310021, China

${ }^{2}$ National Maize Improvement Center of China, Beijing Key Laboratory of

Crop Genetic Improvement, China Agricultural University, Beijing 100193,

China
} 


\section{Background}

Repetitive DNAs, including transposable elements and tandem repeats, are the major components of the genomic sequence and the most important factors in genome size variations in higher eukaryotes [1-3]. Based on the mechanism of transposition, transposable elements can be divided into two classes, transposons and retrotransposons. The majority of these elements in plant genome are long terminal repeat (LTR) retrotransposons and most of them are dispersed throughout all chromosomes $[4,5]$. Tandem repeats consist of large number of repeat units and are usually found in centromeres, pericentromeres or telomeres [6]. Tandem repeats are good cytogenetic markers for chromosome identification and molecular karyotyping [7].

Centromeres are specialized regions on chromosomes where centromeric protein and spindle microtubules attach via the kinetochore and typically contain large arrays of satellite repeats and/or retrotransposon-related repetitive sequences in eukaryotes [8,9]. They are essential for proper chromosome segregation during mitosis and meiosis. Although the function of centromeres is conserved in organisms, centromeric repeats appear to evolve rapidly [10]. Satellite repeats go through rapid evolution and significant variation between closely related species or even among different chromosomes of the same species [11-14]. Centromeric regions are comprised of repetitive sequences in most species, suggesting that those sequences play important roles in centromere function [15]. Centromeres are universally marked by the presence of a centromere-specific histone H3 (CENH3, called CENP-A in human), that replaces canonical histone $\mathrm{H} 3$ in centromeric nucleosomes to form functional centromeres [16]. CENH3 is a good marker to identify the core centromeric sequences by chromatin immunoprecipitation (ChIP) with an anti-CENH3 antibody $[11,17,18]$.

Radish (Raphanus sativus L., $2 \mathrm{n}=2 \mathrm{x}=18$ ), belonging to the family Cruciferae, is an important vegetable crop especially in eastern Asia. Radish root contains various nutritions which play a part in strengthening immunity $[19,20]$. Radish is a healthy vegetable and is popular in many dishes. Although radish is a significant vegetable crop, it still lacks cytogenetic analysis. Location of $5 \mathrm{~S}$ rDNA loci and 45S rDNA loci were confirmed via FISH mapping $[21,22]$. These two sequences are located at the pericentromeric heterochromatin regions. A few studies of the radish repetitive DNAs were previously reported. First an alphoid-like satellite repeat in radish was found in 1986 [23]. It was a big step to get the draft sequences of the Japanese radish 'Aokubi', with a long and thick root, for the study of repetitive elements. It has been estimated that the genome size of the radish is $530 \mathrm{Mb}$ [24] and about $26.6 \%$ of the genome is made of various DNA repeats. The transposons and retrotransposons were characterized [25]. Nevertheless, up to now, understanding of the repetitive sequences of radish is still not sufficient, especially for the tandem repeats. In this study, $5 \mathrm{~Gb}$ of sequence data was used to analyze the repetitive elements of radish. We found three types of tandem repeats (CL1, CL25, and CL43) in the radish genome. An integrated metaphase chromosome karyotype was established using tandem repeats (CL1 and CL25), along with rDNAs as probes. The coding sequence of CENH3 of radish was identified. Immunostaining and chromatin immunoprecipitation tests demonstrated that both CR-Radish and CL1 are associated with RsCENH3 proteins in radish.

\section{Results}

Composition of the repetitive sequences in the radish genome

$5 \mathrm{~Gb}$ sequencing data, which amounts to $4.8 \times$ coverage of the radish genome, was obtained from the HiSeq2000 platform. RepeatExplorer, a Graph-based clustering and characterization of repetitive sequence utilities was used for analyzing repetitive elements of the genome. 174 clusters were generated with cluster size threshold of $0.01 \%$, and clusters which were annotated putative mitochondrial and plastid contaminations were removed. Finally, 144 clusters were used for calculating genome proportions (see Additional file 1). The genome proportions of each type of repetitive DNA are shown in Table 1. About $30.73 \%$ of the genome is repetitive DNAs. According to our results, it has different repetitive DNA types: retrotransposons (including Copia, Gypsy, and LINE/ SINE), transposons (including hAT, Mutator, DNA/CMCEnSpm, and Tc1-Mariner), rDNA and satellites. Satellite repeats, which occupy $12.93 \%$ of the genome, make up the

Table 1 Repeat elements and their proportions in radish

\begin{tabular}{ll}
\hline Elements & GP (genome proportion, \%) \\
\hline Retrotransposon & 11.05 \\
Copia & 5.81 \\
Gypsy & 4.88 \\
LINE/SINE & 0.33 \\
Unclassified LTR & 0.03 \\
Transposon & 1.41 \\
hAT & 0.93 \\
Mutator & 0.27 \\
DNA/CMC-EnSpm & 0.10 \\
Tc1-Mariner & 0.11 \\
rDNA & 4.32 \\
Satellite & 12.93 \\
Unclassified & 1.02 \\
Total & 30.73 \\
\hline
\end{tabular}


most dominant part of the repetitive DNAs in radish. The majority of retrotransposons are Ty1/Copia and Ty3/ Gypsy retrotransposons, with genome proportions of $5.81 \%$ and $4.88 \%$, respectively. The genome proportion of transposons is only $1.41 \%$ and the most abundant transposon is hAT, with a $0.93 \%$ genome proportion. Estimation of rDNA elements abundance showed that they comprise $4.32 \%$ of the genome.

\section{Identification of subtelomeric repeats and centromeric repeats in radish}

In addition to $5 \mathrm{~S}$ rDNA and $45 \mathrm{~S}$ rDNA, three tandemly organized repeats (CL1, CL25 and CL43) were identified by bioinformatics analysis of the sequencing data. The CL1, CL25 and CL43 repeats were estimated to make up $12.32 \%, 0.44 \%$, and $0.17 \%$ of the genome, respectively. CL43 is a telomeric repeat, consisting of a 7 bp monomer (TTTAGGG, the same as the Arabidopsis telomere sequence), located at both ends of chromosomes (see Additional file 2). PCR of CL1 and CL25 resulted in a ladder like pattern for tandemly organized repetitive units. To ascertain the size of the monomers of CL1 and CL25, specific primers were designed for amplifying these two repeats and then sequenced. According to sequencing results, CL1 consists of $\sim 177$ bp monomers (Figure 1), which is almost exactly the same as the size of the alphoid-like satellite repeat reported by Grellet [23]. Searching GenBank and PlantSat databases revealed high similarities to centromeric tandem repeats centBr1 and centBr2 from Brassica species ( $20 \%$ identity over 177 bp) [26] and satellite sequences from Sinapis alba ( 78\% identity over $165 \mathrm{bp}$ ) [27]. The CL25 repeat is characterized by a 348 bp monomer unit (Figure 1) and is a newly found satellite. Similar to CL1, the CL25 sequences shared high similarities to Brassica species $(\sim 78 \%$ identity over $348 \mathrm{bp}$ ). In addition, a small part (the black rectangle outlined region in Figure 1) of the CL25 sequence present in the C. elegans.

FISH result showed that CL1 is located at the main primary constrictions and CL25 appears at the subtelomeric regions (Figure 2a-b). On account of CL25 sharing a high similarity to Brassica species, we speculated that CL25 should have a specific distribution pattern in these species. FISH mapping of CL25 repeats was performed on metaphase chromosomes of several Brassica species, including $B$. rapa (A genome), B. nigra (B genome), $B$. oleracea (C genome), and B. napus (AACC), which are close relatives to radish (Figure 2c-f). Overall, CL25 appeared at subtelomeric regions for all the detected species, although different species have various numbers and varied intensities of signals. Intensities of signals are relatively weak in B. rapa, and strong in B. oleracea. A different distribution pattern was detected in $B$. nigra with strong signals on 4 pairs of chromosomes and weak signals on 2 pairs of chromosomes. Therefore the CL25 repeat is an ancient repeat which appeared before the

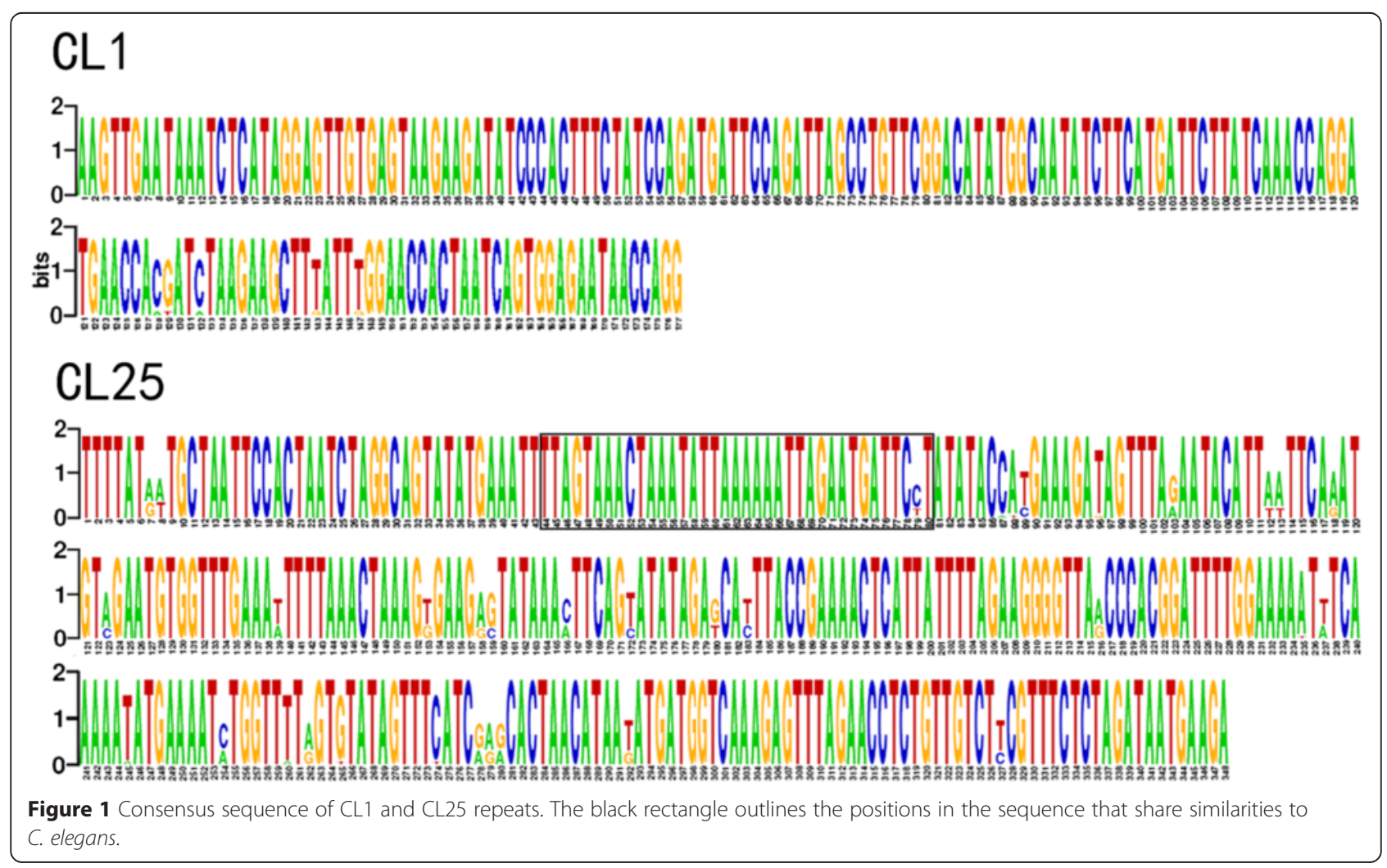



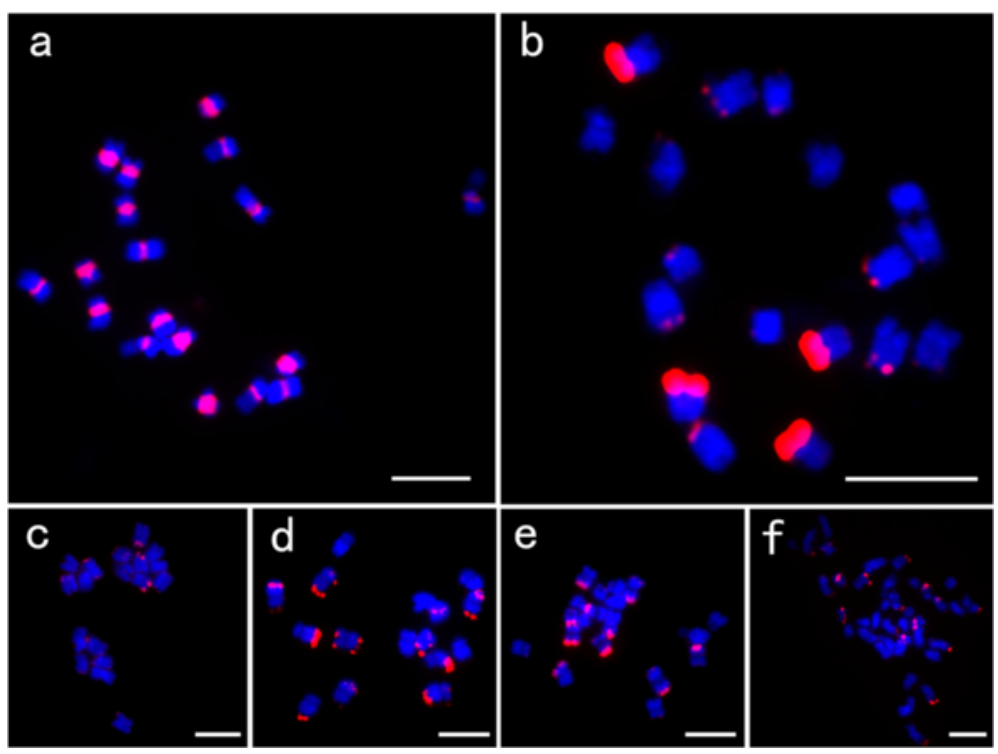

Figure 2 FISH mapping of CL1 and CL25 repeats. (a) FISH mapping of CL1 in radish; (b-f) FISH mapping of CL25; (b) radish; (c) B. rapa; (d) B. nigra; (e) B. oleracea; (f) B. napus. Bars $=5 \mu \mathrm{m}$.

differentiation of tribe Brassiceae and radish. It has maintained its subtelomeric positions in all detected Cruciferae species.

\section{Karyotyping analyses of radish}

Given the lack of DNA markers for FISH analysis, detailed molecular karyotype analyses of radish have not yet been conducted. Repeats identified in this study provide good markers for karyotyping analysis. Sequential FISH using repetitive DNA sequences (CL1, CL25, 5S rDNA and $45 \mathrm{~S}$ rDNA) as probes were performed to identify radish chromosomes (Figure 3a-c). The CL1 signals appeared at the middle of all of the chromosomes with varied intensities. The CL25 signals were located at one arm of chromosomes 1, 4, 5, 6, 7, 8, and 9, and both arms of chromosome 3 with one pairs of signals. Signals were large and strong on chromosomes 5 and 7 , but weak on chromosomes 1, 3, 4, 6, 8 and 9. Two pairs of $5 \mathrm{~S}$ rDNA signals were detected, and one pair of strong signals were located at the peri-centromeric region of the short arm of chromosome 2, and the other pair of weak signals appeared at the peri-centromeric region of the short arm of chromosome 1. Interestingly, we detected 3 pairs of $45 \mathrm{~S}$ rDNA signals in early generations, which is the same as Koo's results [22]; however, only 2 pairs of the signals were detected 3 generations later (see Additional file 3). Seeds from the new generation were used for karyotyping analysis (2 pairs of signals). In our study, the signals of $45 \mathrm{~S}$ rDNA were located at the long arms of chromosomes 2 and 3. Using satellite repeats (CL1 and CL25) combined with rDNAs as FISH probes, we distinctly identified individual somatic chromosome by the position and intensity of their signals (Figure 3d). An integrated ideogram of radish metaphase chromosomes is shown in Figure 3e.

\section{Cloning of CENH3}

To identity CENH3 in radish, we searched NCBI using the blastn program (Nucleotide collection, nr/nt) with the BrCENH3 complementary cDNA sequence (GenBank accession number GU166737.1) as the query. Two radish CENP-A gene sequences (AB299183.1 and AB299184.1) were identified. These two putative CENH3 open reading frames share high similarity with a small gap and some SNPs. Based on these two sequences, specific primers were designed to isolate the complete RsCENH3 coding region from radish plants. According to cDNA sequencing results, three transcripts were detected: a 635 bp length of transcript (1/20), a 513 bp length of transcript (1/20), and the majority $537 \mathrm{bp}$ length of transcript (18/20). To analyze the intron/exon structure of $R s C E N H 3$, the full length of genomic DNA sequence of RsCENH3 was amplified using the same primers. On the basis of genomic DNA results, only one type of DNA sequence was found, which has a total length of $1415 \mathrm{bp}$. This sequence shares $100 \%$ identity to the AB299183.1 and is comprised of nine exons and eight introns. By comparison with the full length genomic DNA sequence, a 635 bp length of transcript transformed from the third intron into an exon, a $513 \mathrm{bp}$ length of the transcript has a deletion from part of the forth exon, and the major transcript is $537 \mathrm{bp}$. Considering the translation, alignment to other plant CENH3s, and the proportion of these transcripts, we deemed that the small number of transcripts were produced by mis- 

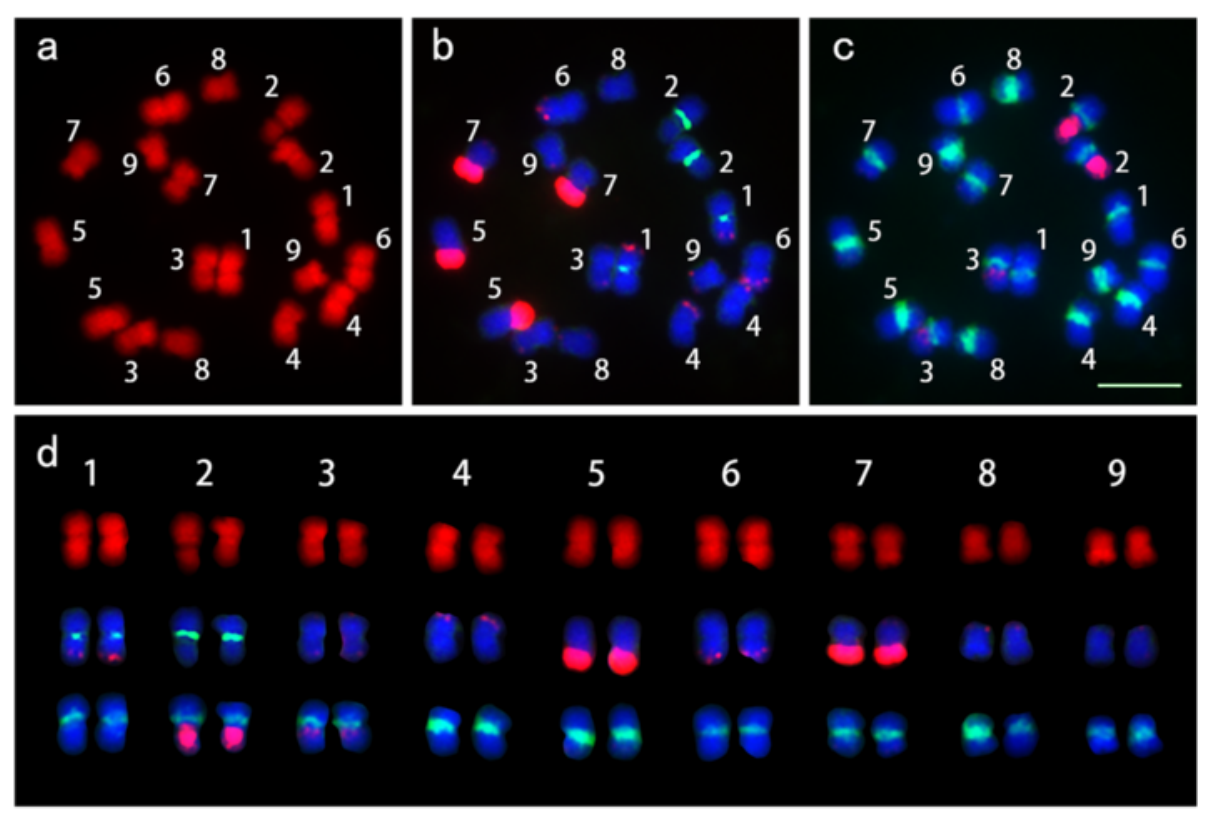

e
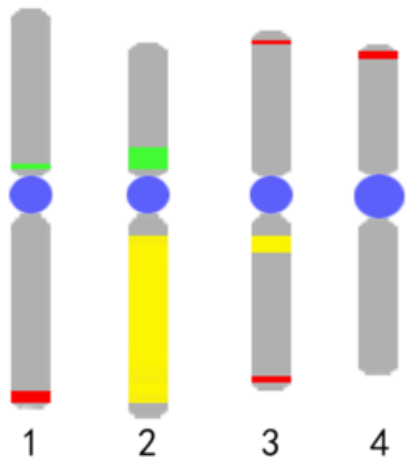

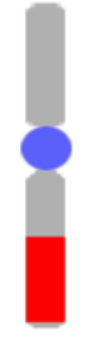

4

\section{5}

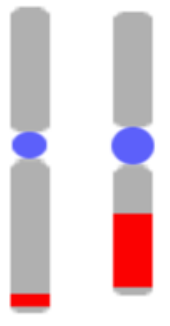

6

7

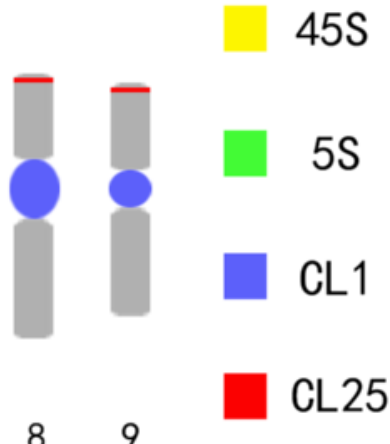

Figure 3 The karyotype and ideograph for radish mitotic metaphase chromosomes. (a) The mitotic metaphase chromosomes (numbered from 1 to 9) were counterstained with DAPI and pseudocolored in red; (b) FISH with the probe of CL25 (red) and 5S rDNA (green); (c). The same spread was reprobed with the probe of CL1 (green) and 45S rDNA (red); (d) Individual chromosomes were separated from Figure (a-c) and listed according to their order; (e) Ideogram showing the position and intensity of CL25 (red) and 5S rDNA (green), CL1 (blue) and 45S rDNA (yellow). Bars $=5 \mu \mathrm{m}$.

splicing from the same loci and the CENH3 comprises an open reading frame (ORF) of length 537 bp encoding a predicted 178-amino acid (Aa) protein.

Multiple sequence alignment revealed that RsCENH3 shares high identities with CENH3 from Brassica species, 77\% identity with BrCENH3, 64\% with BnCENH3, and $74 \%$ with BoCENH3. Several prominent features of the deduced RsCENH3 in comparison with those CENH3s and canonical histone $\mathrm{H} 3$ are as follows (Figure 4). A longer and more divergent $\mathrm{N}$-terminal tail is present in the deduced RsCENH3 sequence (178 amino acids in total) that is not alignable to $\mathrm{BrH} 3$ (136 amino acids in total). Each of the predicted proteins encoded a histone fold domain with similarities to histone $\mathrm{H} 3$. The loop 1 region in the histone fold domain is longer than that of canonical histone H3s (nine amino acids as opposed to seven for BrH3). All of these findings demonstrate that the sequence identified is an authentic CENH3 homolog in radish.

\section{DNA sequences associated with RsCENH3}

A B. rapa -derived CENH3 antibody (anti-BrCENH3) was previously used to confirm CENH3-associated centromeric sequences in different Brassica species [28]. Based on the similarities of CENH3's sequence between radish and Brassica species, we speculated B. rapa -derived CENH3 antibody should recognize the $\mathrm{RsCENH} 3$ protein at core centromeres in radish. To confirm whether it recognizes the RsCENH3 protein, we applied an immunofluorescence assay on somatic cells of radish with the anti-BrCENH3 antibody. Signals appeared at the centromeric regions of 


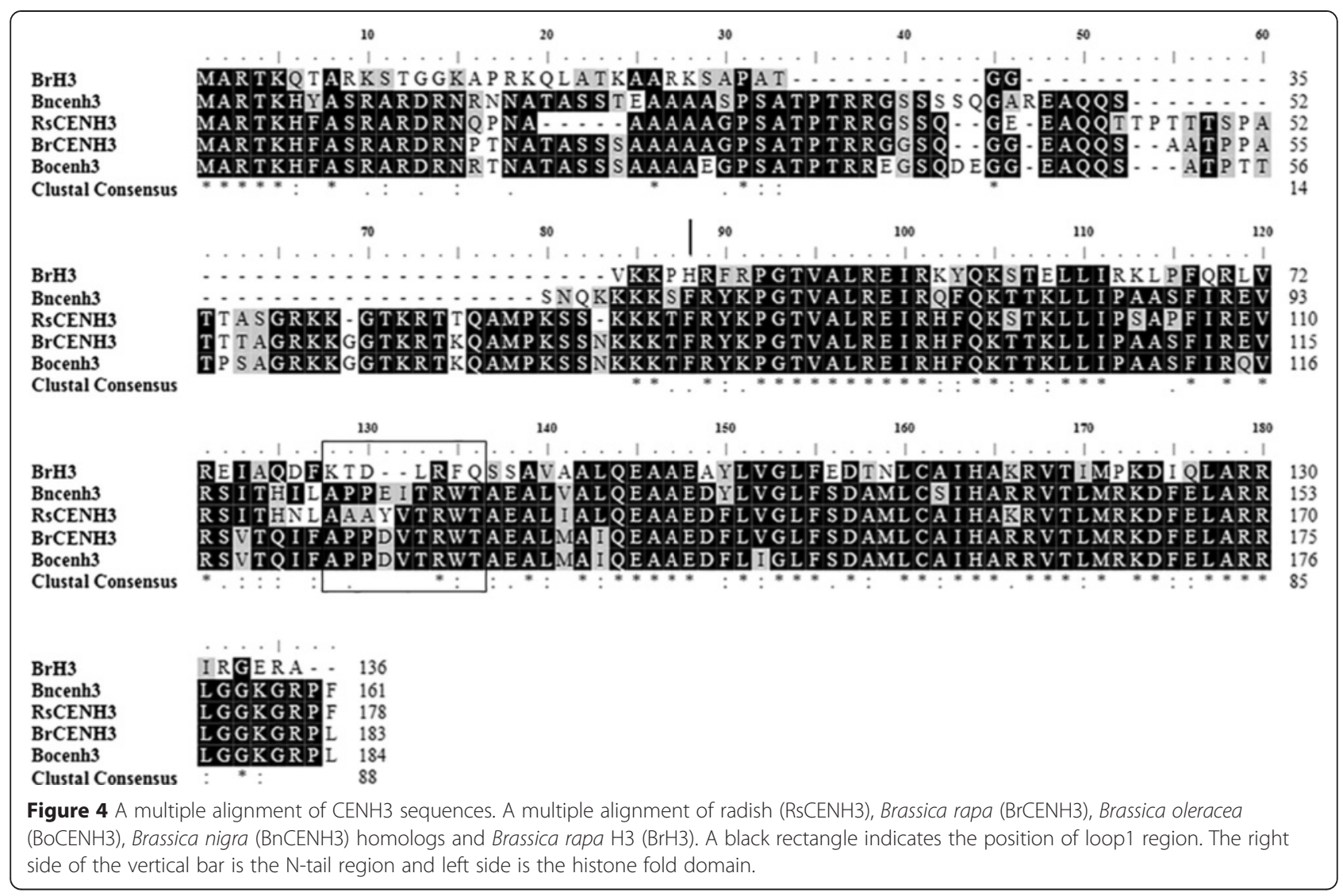

all 18 metaphase chromosomes (Figure 5d-f). In interphase cells, RsCENH3 signals were located at the edge of the DAPI intensively stained heterochromatic regions (Figure 5a-c). It showed that the antibody also could recognize RsCENH3.

It has been reported that CRB (Centromere-specific retrotransposons of Brassica) is a core centromeric sequences of Brassica species [28]. We also detected a CRBlike retrotransposon CL4, which represents $1.14 \%$ of the genome and was named CR-Radish in radish. To verify if the centromere-specific retrotransposon CR-Radish and the 177-bp satellite repeat CL1 were associated with RsCENH3 protein in radish, we performed an immunofluorescence assay followed by FISH on the same set of cells to detect the co-localization of BrCENH3 and centromeric DNA repeats. The size of RsCENH3 immunosignals were relatively uniform among kinetochores while the size of CL1 signals were uneven among different chromosomes (Figure 6a-d). CL1 signals were overlapped with the RsCENH3 immuno-signals, although they were significantly larger than the RsCENH3 immuno-signals. These results suggest that only a limited part of the CL1 sequences are associated with the kinetochore complex. We also conducted anti-BrCENH3 immunostaining followed by FISH of the CR-Radish retrotransposon
(Figure 6e-h). Different from that of CL1, CR-Radish signals were smeared and weak. As expected, the FISH signals overlapped with most of the immuno-signals. Therefore, we propose that the RsCENH3 protein is also associated with CR-Radish. Dual-color FISH showed most signals of CR-Radish and CL1 were co-localized, while the signals of CL1 were more concentrated than CR-Radish signals (Figure 6i-1).

To further confirm our immunostaining results, ChIP tests with the anti-BrCENH3 antibody were conducted to assess the association of CL1 and CR-Radish with RsCENH3. FISH using the ChIPed DNA as a probe showed high enhanced signals in the centromere regions of all radish chromosomes. In contrast, using mocked DNA as a probe showed no obvious signal (see Additional file 4) which indicates that the centromere sequences were specifically pulled down by the anti-BrCENH3 antibody in ChIP. The ChIP-qPCR was performed to verify the enrichment of putative centromeric repeats (Figure 7). Two specific primers, designed from different regions of CL1, CL25 and CR-Radish, were used to detect each fragment. The ChIP-qPCR was repeated three times using CL25 as extra-centromeric control. RFE value for CL25-1 was set at 1 , and the RFE value of each sequence was normalized using the CL25-1 as a reference. The RFE of the non- 

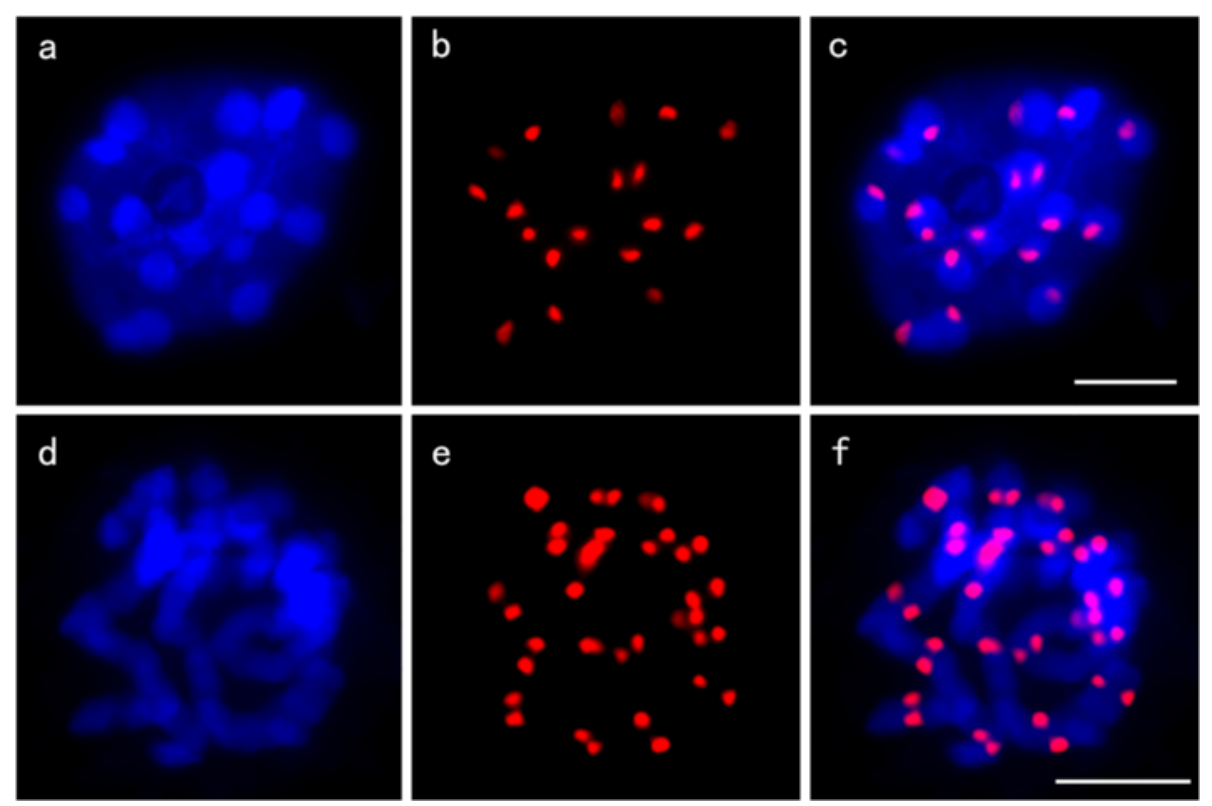

Figure 5 Anti-BrCENH3 antibody staining in mitotic cells of radish. RsCENH3 localization (red) on somatic interphase cell (a-c) and metaphase chromosomes (d-f). Bars $=5 \mu \mathrm{m}$.

centromeric control CL25-2, 5S rDNA, and 45S rDNA were low and similar to each other at $1.06 \pm 0.04,1.11 \pm$ 0.03 , and $1.41 \pm 0.02$, respectively (Figure 7 ). In contrast, the RFE of the CR-Radish fragments were as high as $22.02 \pm 0.94$ and $18.54 \pm 0.53$, respectively. Similarly, the RFE of the CL1 fragments were $13.71 \pm 0.33$ and $11.64 \pm$ 0.11 , respectively. These results indicate that CR-Radish and CL1 were significantly enriched in the ChIPed DNA. Therefore, CR-Radish and CL1 are associated with RaCENH3.

\section{Discussion}

\section{Karyotype of radish}

Up to now, studies on the radish genome were still limited and few cytogenetic and genomic studies were carried out [21,22,25]. Comparative analysis of rDNA and Rfk1 gene distribution in chromosomes of Brassica species and radish were carried through using FISH $[21,22,29]$. However, to our knowledge, a complete karyotype analysis that reliably distinguishes each chromosome of radish has not been reported. Chromosome identification is critical for cytological analyses, as well as subsequent studies in genomics, taxonomy, and the evolution of polyploidy, enabling an understanding of the relationship between visible landmarks and genetic or physical map features [30]. The somatic metaphase chromosomes of radish are small and lack feasible markers, which make adequate identification of radish chromosome pairs difficult. In this study, we used RepeatExplorer to conduct genome-wide analysis of repetitive sequences and obtained two useful cytogenetic markers (CL1 and CL25).
Together with rDNAs, one or two signals were detected on each chromosome (Figure $3 \mathrm{~d}$ ). We are now able to easily identify all 9 somatic metaphase chromosomes by the position and intensity of FISH signals. In addition, an integrated metaphase chromosome karyotype was established (Figure 3e). Our study provides a valuable basis for future genomic studies.

\section{Dynamic nature of radish genome}

Repetitive sequences contribute significantly to extraordinary genome size variation in higher plants [31,32]. Generally speaking, LTR-retrotransposons are the most abundant element of the genome, especially in big genome species, such as maize [5], wheat [33], and coix [34]. However, the majority of repetitive sequences are satellites, which make up $12.932 \%$ of the radish genome in our study. A similar high proportion of satellites were found in C. rubella and cucumber, in which more than $20 \%$ of the genome sequences are satellite repeats $[35,36]$. Ordinarily, several to dozens of types of satellite repeats are detected from a number of species [34,37-39]. In our study, only three satellite repeats were found in radish, including centromeric repeats, subtelomeric repeats and telomeric repeats (Figure 3). This is a typical pattern where the satellite DNA sequences are appear predominantly in the centromeric, pericentromeric and telomeric regions [40,41]. The dynamic evolutionary processes of satellite DNA may generate changes in its chromosomal location and distribution. Some satellite DNA families were found to be species-specific [42], while others were 


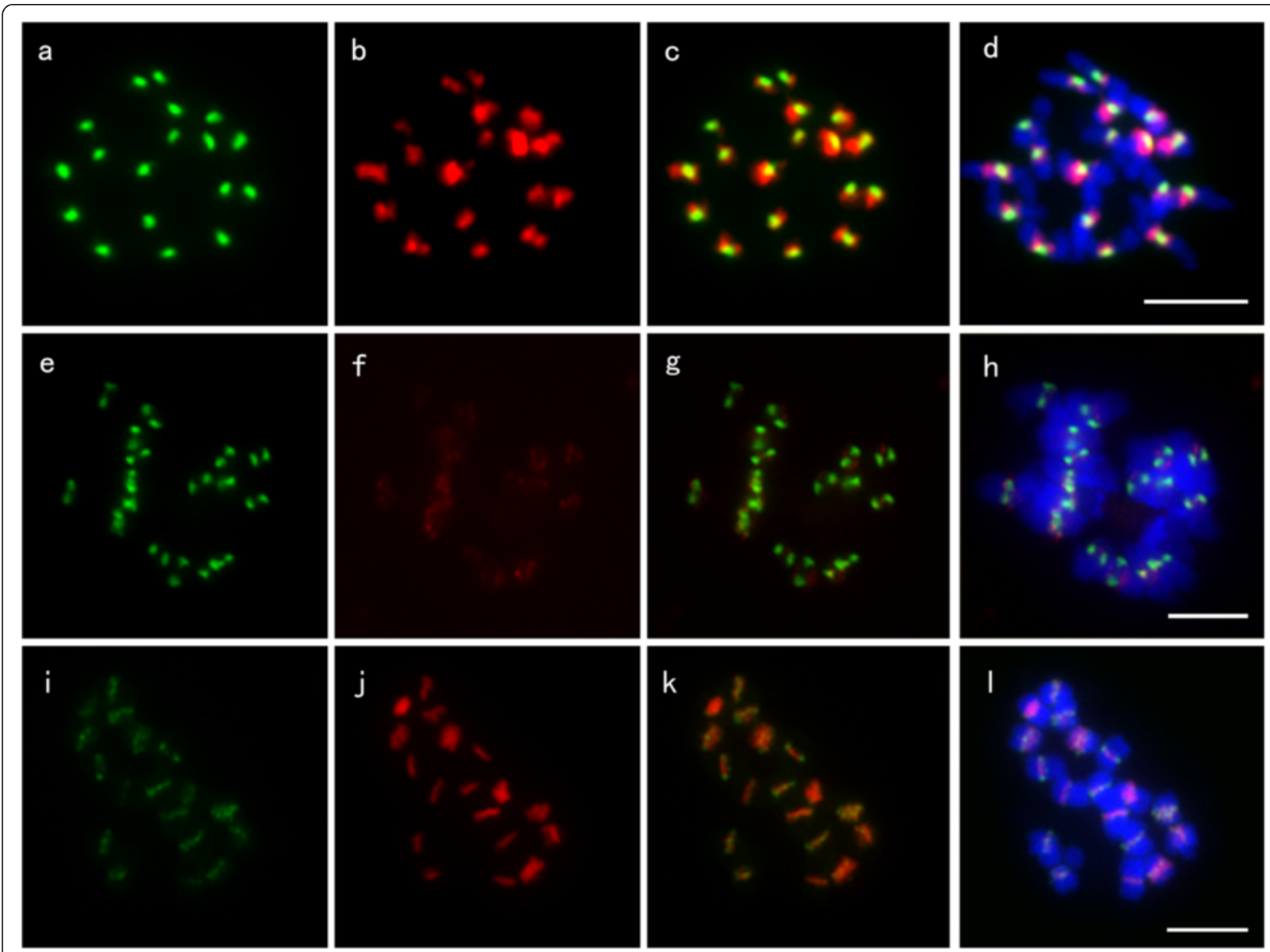

Figure 6 Sequential localization of the anti-BrCENH3 antibody and centromeric repeats on radish. (a) RsCENH3 localization at mitotic metaphase chromosomes of radish; (b) The same cell was hybridized with CL1; (c) Merged fluorescence signals from a and b; (d) Merged fluorescence signals from c and chromosomes; (e) RsCENH3 localization at mitotic metaphase chromosomes of radish; (f) The same cell was hybridized with CR-Radish; (g) Merged fluorescence signals from d and e; (h) Merged fluorescence signals from g and chromosomes; (i) CR-Radish localization at mitotic metaphase chromosomes of radish; (j) The same cell was probed with CL1; (k) Merged fluorescence signals from $g$ and $h$; (I) Merged fluorescence signals from $\mathrm{h}$ and chromosomes. Bars $=5 \mu \mathrm{m}$.

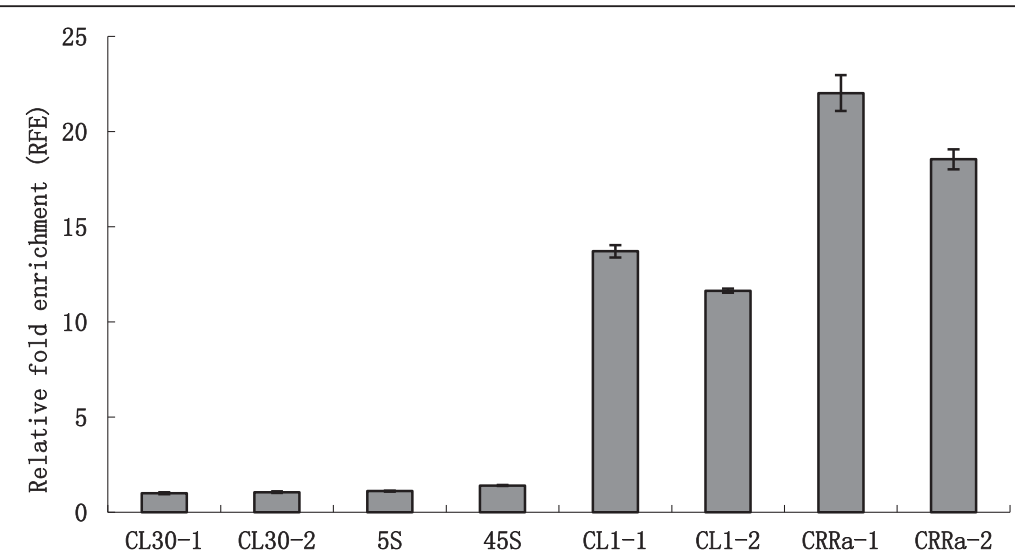

Figure 7 Sequences associated with RsCENH3. Relative fold enrichments of repeats obtained by ChIP with the anti-BrCENH3 antibody are shown for radish genomes. CL25 serves as a negative control; CR-Radish and CL1 were associated with CENH3. 
more conserved, and similar sequences may be isolated in closely related species [26,43]. In our study, we detected 3 pairs of $45 \mathrm{~S}$ rDNA signals in early generations of the radish, the same result obtained by Koo [22], while only 2 pairs of $45 \mathrm{~S}$ rDNA signals were detected in later generations (see Additional file 3 ). It suggests that rDNA also have a rapid evolution in the genome. Furthermore radish inbred lines from different areas might contain varied ratio of repetitive sequences. $30.73 \%$ of the $0713 \mathrm{D}$ genome is repetitive DNA in our study, while repetitive sequences occupied $26.6 \%$ of the Japanese radish 'Aokubi' genome [25].Compositions of each type of repetitive elements are also different between these two radishes. Overall, these results demonstrate the highly dynamic nature of radish genome.

\section{Rapid evolution of centromere sequence}

The centromeres of higher eukaryotes are rich in repetitive DNA sequences which include large arrays of satellite repeats and/or retrotransposon-related repetitive sequences $[8,9]$. It has been shown that one single major satellite repeat is the dominating sequence in all centromeres in most diploid species [8,9]. In our study, the similar pattern of one type of centromeric satellite repeat (CL1) was detected by immunostaining and the ChIP test. However, it has been reported that some plant and animal species contain multiple satellite repeats associated with centromeres, such as in the common bean [44], potato [13], and chicken [45]. Centromeric satellite repeats diverge rapidly across species and often do not share any sequence similarity [8]. Several centromeric repeats were identified in potato and its closely related wide species $S$. verrucosum, respectively. Nevertheless, only one single homoeologous centromeric sequence was detected between these two species. This means centromeric regions of Solanum species show rapid evolution.

Taxonomic studies and rDNA gene space sequence analysis demonstrated that genus Brassica is a close relative of the genus Raphanus [46,47]. Our results also proved this. In this study, a new satellite CL25 was detected, which is distributed in radish and all tested Brassica species and located at the subtelomeric region of all tested species (Figure 2). Even in closely related species, centromeric satellites go through rapid evolution. CL1, the centromeric satellite repeat, shares high similarities with $\mathrm{CentBr} 1$ and $\mathrm{CentBr} 2$ sequences. These $\mathrm{CentBr}$ sequences appeared in the $\mathrm{A}$ and $\mathrm{C}$ genomes of Brassica species, while the corresponding centromeric repeats have not yet been identified in the B genome. Even in the same species, CentBr1 and CentBr2 have different distribution patterns on chromosomes [26]. These results indicate that centromeric satellite repeats of Cruciferae species evolve rapidly.

\section{Conclusions}

In this study, we used low-coverage sequencing on Raphanus sativus $\mathrm{L} . \quad(2 \mathrm{n}=18)$ to analyze repeat elements. We revealed the genome structure of radish and found that satellite repeats are most dominating elements, which is differ from most reported species, in which LTRretrotransposons are the most abundant element of the genome. The fine karyotyping analysis using satellites and rDNAs as markers allow us to easily identify each individual somatic metaphase chromosome. Only one putative CENH3 (RsCENH3) gene was characterized and cloned from radish. Its deduced amino acid sequence shares high similarities to those of the CENH3s in Brassica species. In addition, Immunofluorescence- and ChIP-based assays demonstrated the functional significance of satellite and centromere-specific retrotransposon at centromeres. Our study provides a valuable basis for future genomic studies in radish.

\section{Availability of supporting data}

The data sets supporting the results of this article are available in the NCBI SRA archive (accession no. SRX957720).

\section{Methods}

\section{Plant materials}

0713D ( $2 \mathrm{n}=2 \mathrm{x}=18, \mathrm{R}$ genome), a Chinese Raphanus sativus L. inbred line, was used for Solexa genome sequencing, ChIP and cytogenetic studies. Plants were grown in the greenhouse with 16 hours in lights and 8 hours in the dark.

\section{Genomic DNA isolation and Solexa sequencing}

DNA was isolated from $5 \mathrm{~g}$ of fresh young plant as described previously [48]. DNA was treated with DNase-freeRNase A for $3 \mathrm{~h}$ at RT for removing RNA, and purified by phenol/chloroform precipitation. Pellets were resuspended to a final concentration of 200-300 ng/ $\mu \mathrm{l}$. The sequencing was performed by HiSeq2000 platform (BerryGenomics. Beijing, China). One hundred bp paired-end reads were obtained from the results.

\section{Data analysis}

Following a removal of linker/primer contaminations and artificially duplicated reads, a set of $5 \mathrm{~Gb}$ whole genome Illumina paired end reads (Average length of reads was $100 \mathrm{bp}$ ), representing about $4.8 \times$ genome equivalent of radish [24] were used for similarity-based clustering analysis [38]. The clustering analysis was performed using a read similarity cutoff of $90 \%$ over at least $70 \%$ of the shorter sequence length. Reads within individual clusters were assembled into contigs. Sequence-similarity searches of assembled contigs were done for finding out which type and family of repeats they present. Clusters containing satellite repeats were identified based on graphs and the 
presence of tandem repeats within assembled contig sequences. Satellite sequences were identified using the Tandem Repeat Finder [49]. Clusters corresponding to putative mitochondrial and plastid contaminations were identified by searching GenBank and eliminated. The genome proportion of each cluster was calculated as the percentage of reads.

\section{FISH and immunostaining}

In the FISH procedure, mitotic chromosomes were prepared as follows: seeds were geminated on moist miracloth at $28^{\circ} \mathrm{C}$ in the dark for 2 days, root tips from radish were collected and treated with pressurized nitrous oxide for 90 min, fixed in 3:1 (100\% ethanol: glacial acetic acid) Carnoy's solution for 2 days at room temperature $\left(25^{\circ} \mathrm{C}\right)$ and then stored at $-20^{\circ} \mathrm{C}$ until used. Probes were prepared by PCR amplification from radish genomic DNA with specific primers (see Additional file 5). The amplified DNAs were labeled with bio-16-UTP, digoxigenin-11-dUTP or DEAC (Roche. Basel, Switzerland) using a standard nick translation reaction. The FISH experiments, including slide pre-treatment, probe hybridization and signal detection were performed as reported according to published protocols [17]. Chromosomes were counterstained with 4', 6-diamidino-2-phenylindole (DAPI) (Vector Laboratories. Burlingame, USA). Images were captured digitally using a Sensys CCD camera (QIMAGING, RETIGA-SRV, FAST 1394) attached to an Olympus BX61 epifluorescence microscope (Olympus. Tokyo, Japan). Images were adjusted with Adobe Photoshop 5.0. In order to draw an integrated ideogram of radish metaphase chromosomes, chromosomes in 5 metaphase cells were measured.

In the immunostaining procedure, root tips were fixed in freshly prepared $4 \%(\mathrm{w} / \mathrm{v})$ paraformaldehyde solution for $30 \mathrm{~min}$ on ice and then washed three times for $10 \mathrm{~min}$ in $1 \times$ PBS $(10 \mathrm{mM}$ sodium phosphate, $\mathrm{pH} 7.0$, and $140 \mathrm{mM}$ $\mathrm{NaCl})$ on ice. After washing with $1 \times \mathrm{PBS}$, the root tips were directly squashed on slides coated with poly-L-lysine. After removal of the cover slip, the slides were immersed in $1 \times$ PBS. The slides were incubated for $3 \mathrm{~h}$ at $37^{\circ} \mathrm{C}$ in a moist chamber with the mouse primary sera antibody against brassica CENH3 diluted in $1 \times$ TNB buffer. Following three rounds of washing in $1 \times$ PBS, anti-mouse-Alexa 488 diluted in 1:100 was applied for $1 \mathrm{~h}$ at $37^{\circ} \mathrm{C}$. After three rounds of washing in $1 \times \mathrm{PBS}$, the slides were dried at room temperature. For detection of the CENH3 proteins, the chromosomes were counterstained with DAPI. For a combined detection of the CENH3 proteins and the satellite repeats, the slides were fixed in $4 \%(\mathrm{w} / \mathrm{v})$ paraformaldehyde solution for $5 \mathrm{~min}$ and washed in $1 \times$ PBS for three times, then the FISH procedure was followed as usual.

\section{ChIP and quantitative ChIP-PCR}

ChIP using the BrCENH3 antibody was performed on radish nucleosomes as previously described [50]. Approximately
$10 \mathrm{~g}$ of 10-days-old radish plants were used for isolating nuclei. The isolated nuclei were suspended in $3 \mathrm{ml}$ micrococcal nuclease (MNase) buffer (10\% sucrose, $50 \mathrm{mM}$ Tris- $\mathrm{HCl} \mathrm{Ph} \mathrm{7.5,} 4 \mathrm{mM} \mathrm{MgCl}$, and $\left.1 \mathrm{mM} \mathrm{CaCl}_{2}\right)$ and then digested with micrococcal nuclease (Sigma) to produce a chromatin solution. The digested chromatin was used for ChIP experiments using the BrCENH3 antibody, and normal mouse serum was used as a mock treatment. Chromatin with the antibody was incubated with rotation overnight at $4{ }^{\circ} \mathrm{C}$. DNA from the ChIP and input control samples was diluted in $1 \times \mathrm{TE}$.

Quantitative real-time PCR analysis of pelleted DNA was used to determine the relative fold enrichment (RFE) of specific sequences within anti-BrCENH3 precipitated DNA relative to the DNA sample prepared from preblood immunoprecipitation. We used the CL25, which is located at the chromosome ends, as a negative control to normalize enrichment of each positive amplicon. Each sample had three replicates. 5S rDNA and 45S rDNA, which were not localized at centromere region, were also used for evaluating reliability of the results. Primers CL251L, CL25-1R, CL25-2L, CL25-2R, 5SL, 5SR, 45SL, 45SR, CL1-1L, CL1-1R, CL1-2L, CL1-2R, CR-Radish-1L, CRRadish-1R, CR-Radish-2L and CR-Radish-2R were used for real-time PCR and are listed in Additional file 1: Table S2. The relative expression levels were calculated according to cycle number. Quantitative PCR data were performed as described previously [28].

\section{Cloning of CENH3 CDNA}

To identify radish CENH3 orthologs sequences, the $\mathrm{BrCENH} 3$ complementary cDNA sequence (GenBank accession number GU166737.1), as the query, was searched by NCBI BLAST. Two radish CENP-A genes sequence were identified. Total RNA was extracted from leaf tissue of an inbred line '0713D'. RNA samples were treated with RNase-free DNase (Promega. Madison, USA) and dissolved in RNase-free double-distilled water. cDNA was synthesized using the RNA and Superscript III RT (Invitrogen, Carlsbad, USA). The primers CENH3-L and CENH3-R were used for amplification of full length CDS of CENH3. The fragments were cloned and sequenced. Multiple sequence alignment of CENH3 was performed using MUSCLE [51].

\section{Additional files}

Additional file 1: List of the annotation and genome proportion of clusters.

Additional file 2: FISH mapping of CL43 repeats in radish.

Additional file 3: FISH mapping of 45S rDNA in different

generation of radish. (a) Early generation; (b) Later generation. 
Additional file 4: ChIP-FISH using BrCENH3 antibodies. (a) FISH signals derived from ChIP using normal mouse serum; (b) FISH signals derived from ChIP using anti-CENH3 antibodies.

\section{Additional file 5: Primers used in this study.}

\section{Abbreviations}

FISH: Fluorescent in situ hybridization; Gb: Giga base pairs; Mb: Mega base pairs; bp: Base pair; ChIP: Chromatin immunoprecipitation.

\section{Competing interests}

The authors declare that they have no competing interests.

\section{Authors' contributions}

$\mathrm{QH}$ carried out molecular, cytogenetic studies and drafted the manuscript. ZC carried out bioinformatics analysis, participated in the ChIP assay and provided helpful discussions. TH helped to maintain the experiment materials. $\mathrm{CB}$ and $\mathrm{HL}$ participated in cytogenetic studies. WJ and WM designed the experiment and revised the manuscript. All authors read and approved the final manuscript.

\section{Acknowledgments}

This research was supported by National Science Foundation of China (31171563) and the Grand Science and Technology Special Project of Zhejiang Province (2012C12903-3-6). Authors also thank Dr. Kyle Q. Hoffmann for linguistic advice in the preparation of this manuscript.

\section{Received: 2 January 2015 Accepted: 23 March 2015}

\section{Published online: 18 April 2015}

\section{References}

1. Kumar A, Bennetzen JL. Plant retrotransposons. Annu Rev Genet. 1999:33:479-532.

2. Bennetzen $J$, Ma J, Devos KM. Mechanisms of recent genome size variation in flowering plants. Ann Bot. 2005:95:127-32

3. Lysak MA, Koch MA, Beaulieu JM, Meister A, Leitch IJ. The dynamic ups and downs of genome size evolution in Brassicaceae. Mol Biol Evol. 2009;26:85-98.

4. Arabidopsis Genome Initiative. Analysis of the genome sequence of the flowering plant Arabidopsis thaliana. Nature. 2000;408:796-815.

5. Maize Genome Initiative. The B73 maize genome: complexity, diversity, and dynamics. Science. 2009;326:1112-5.

6. Sharma S, Raina SN. Organization and evolution of highly repeated satellite DNA sequences in plant chromosomes. Cytogenet Genome Res. 2005;109:15-26.

7. Kato A, Vega JM, Han F, Lamb JC, Birchler JA. Advances in plant chromosome identification and cytogenetic techniques. Curr Opin Plant Biol. 2005;8:148-54.

8. Henikoff S. Chromosomes on the move. Trends Genet. 2001;17:689-90.

9. Jiang J, Birchler JA, Parrott WA, Dawe RK. A molecular view of plant centromeres. Trends Plant Sci. 2003:8:570-5.

10. Melters DP, Bradnam KR, Young HA, Telis N, May MR, Ruby JG, et al. Comparative analysis of tandem repeats from hundreds of species reveals unique insights into centromere evolution. Genome Biol. 2013;14:R10.

11. Zhong CX, Marshall JB, Topp C, Mroczek R, Kato A, Nagaki K, et al. Centromeric retroelements and satellites interact with maize kinetochore protein CENH3. Plant Cell. 2002;14:2825-36.

12. Wang G, Zhang X, Jin W. An overview of plant centromeres. J Genet Genomics. 2009;36:529-37.

13. Gong Z, Wu Y, Koblízková A, Torres GA, Wang K, lovene M, et al. Repeatless and repeat-based centromeres in potato: implications for centromere evolution. Plant Cell. 2012;24:3559-74.

14. Zhang H, Kobližková A, Wang K, Gong Z, Oliveira L, Torres GA, et al. Boom-bust turnovers of megabase-sized centromeric DNA in Solanum species: rapid evolution of DNA sequences associated with centromeres. Plant Cell. 2014;26:1436-47.

15. Fukagawa T, Earnshaw WC. The centromere: chromatin foundation for the kinetochore machinery. Dev Cell. 2014;30:496-508.

16. Malik HS, Henikoff S. Major evolutionary transitions in centromere complexity. Cell. 2009;138:1067-82.
17. Jin W, Melo JR, Nagaki K, Talbert PB, Henikoff S, Dawe RK, et al. Maize centromeres: organization and functional adaptation in the genetic background of oat. Plant Cell. 2004;16:571-81.

18. Houben A, Schroeder-Reiter E, Nagaki K, Nasuda S, Wanner G, Murata M, et al. CENH3 interacts with the centromeric retrotransposon cereba and GC-rich satellites and locates to centromeric substructures in barley. Chromosoma. 2007;116:275-83

19. Uda Y, Hayashi $H$, Shimizu A. Mutagenic andanti-mutagenic property of 3-hydroxymethylene-2-thioxopyrrolidine, a major product generating from pungent principle of radish. Lebensm Wiss Technol. 2000;33:37-43.

20. Yamasaki M, Omi Y, Fujii N, Ozaki A, Nakama A, Sakakibara Y, et al. Mustard oil in 'shibori daikon' a variety of Japanese radish selectively inhibits the proliferation of H-ras-transformed 3Y1 cells. Biosci Biotechnol Biochem. 2009;73:2217-21.

21. Hasterok R, Wolny E, Hosiawa M, Kowalczyk M, Kulak-ksiazczyk S, Ksiazyk T, et al. Comparative analysis of rDNA distribution in chromosomes of various species of Brassicaceae. Ann Bot. 2006:97:205-16.

22. Koo DH, Hong CP, Batley J, Chung YS, Edwards D, Bang JW, et al. Rapid divergence of repetitive DNAs in Brassica relatives. Genomics. 2011;97:173-85.

23. Grellet F, Delcasso D, Panabieres F, Delseny M. Organization and evolution of a higher plant alphoid-like satellite DNA sequence. J Mol Biol. 1986;187:495-507.

24. Marie D, Brown SC. A cytometric exercise in plant DNA histograms, with 2C values for 70 species. Biol Cell. 1993;78:41-51.

25. Kitashiba H, Li F, Hirakawa H, Kawanabe T, Zou ZW, Hasegawa Y, et al. Draft sequences of the radish (Raphanus sativus L.) genome. DNA Res. 2014;21:481-90

26. Lim KB, Yang TJ, Hwang YJ, Kim JS, Park JY, Kwon SJ, et al. Characterization of the centromere and peri-centromere retrotransposons in Brassica rapa and their distribution in related Brassica species. Plant J. 2007:49:173-83.

27. Capesius I. Sequence of the cryptic satellite DNA from the plant Sinapis alba. Biochim Biophys Acta. 1983;739:276-80

28. Wang GX, He QY, Liu F, Cheng ZK, Talbert PB, Jin WW. Characterization of CENH3 proteins and centromere-associated DNA sequences in diploid and allotetraploid Brassica species. Chromosoma. 2011;120:353-65.

29. Niemelä T, Seppänen M, Badakshi F, Rokka V, Heslop-Harrison JS. Size and location of radish chromosome regions carrying the fertility restorer Rfk 1 gene in spring turnip rape. Chromosome Res. 2012;20:353-61.

30. Harper LC, Cande WZ. Mapping a new frontier; development of integrated cytogenetic maps in plants. Funct Integr Genomics. 2000;1:89-98.

31. Piegu B, Guyot R, Picault N, Roulin A, Saniyal A, Kim H, et al. Doubling genome size without polyploidization: Dynamics of retrotransposition driven genomic expansions in Oryza australiensis, a wild relative of rice. Genome Res. 2006;16:1262-9.

32. Hawkins JS, Kim H, Nason JD, Wing RA, Wendel JF. Differential lineage-specific amplification of transposable elements is responsible for genome size variation in Gossypium. Genome Res. 2006:16:1252-61.

33. International Wheat Genome Sequencing Consortium. Aegilops tauschii draft genome sequence reveals a gene repertoire for wheat adaptation. Nature. 2013;496:91-5

34. Cai ZX, Liu HJ, He QY, Pu MW, Chen J, Lai JS, et al. Differential genome evolution and speciation of Coix lacryma-jobi L. and Coix aquatica Roxb. hybrid guangxi revealed by repetitive sequence analysis and fine karyotyping. BMC Genomics. 2014;15:1025.

35. Han YH, Zhang ZH, Liu JH, Lu JY, Huang SW, Jin WW. Distribution of the tandem repeat sequences and karyotyping in cucumber (Cucumis sativus L.) by fluorescence in situ hybridization. Cytogenet Genome Res. 2008;122:80-8.

36. Slotte T, Hazzouri KM, Ågren JA, Koenig D, Maumus F, Guo YL, et al. The Capsella rubella genome and the genomic consequences of rapid mating system evolution. Nat Genet. 2013;45:831-5.

37. Hnibová 1E, Neumann P, Matsumoto T, Roux N, Macas J, Dolezel J. Repetitive part of the banana (Musa acuminata) genome investigated by low-depth 454 sequencing. BMC Plant Biol. 2010;10:204

38. Macas J, Neumann P, Navrátilová A. Repetitive DNA in the pea (Pisum sativum L.) genome: comprehensive characterization using 454 sequencing and comparison to soybean and Medicago truncatula. BMC Genomics. 2007:8:427.

39. Piednoe"l M, Aberer AJ, Schneews GM, Macas J, Novak P, Gundlach H, et al. Next-generation sequencing reveals the impact of repetitive DNA across phylogenetically closely related genomes of Orobanchaceae. Mol Biol Evol. 2012;29:3601-11. 
40. Charlesworth B, Sniegowski P, Stephan W. The evolutionary dynamics of repetitive DNA in eukaryotes. Nature. 1994;371:215-20.

41. López-Flores I, Ramos-Garrido MA. The repetitive DNA content of eukaryotic genomes. Genome Dyn. 2012;7:1-28.

42. Kopecna O, Kubickova S, Cernohorska H, Cabelova K, Vahala J, Rubes J. Isolation and comparison of tribe-specific centromeric repeats within Bovidae. J Appl Genet. 2012;53:193-202.

43. Torres GA, Gong Z, Lovene M, Hirsch CD, Buell CR, Bryan GJ, et al. Organization and evolution of subtelomeric satellite repeats in the potato genome. G3 (Bethesda). 2011;1:85-92.

44. Iwata A, Tek AL, Richard MM, Abernathy B, Fonsêca A, Schmutz J, et al. Identification and characterization of functional centromeres of the common bean. Plant J. 2013;76:47-60.

45. Shang WH, Hori T, Toyoda A, Kato J, Popendorf K, Sakakibara Y, et al. Chickens possess centromeres with both extended tandem repeats and short non-tandem-repetitive sequences. Genome Res. 2010;20:1219-28.

46. Lü N, Yamane K, Ohnishi O. Genetic diversity of cultivated and wild radish and phylogenetic relationships among Raphanus and Brassica species revealed by the analysis of trnK/matK sequence. Breed Sci. 2008;58:15-22.

47. Mun JH, Kwon S, Yang T, Seol Y, Jin M, Kim J, et al. Genome-wide comparative analysis of the Brassica rapa gene space reveals genome shrinkage and differential loss of duplicated genes after whole genome triplication. Genome Biol. 2009;10:R111.

48. Doyle JJ. A rapid DNA isolation procedure for small quantities of fresh leaf tissue. Phytochem Bull. 1987;19:11-5.

49. Benson G. Tandem Repeat Finder: a program to analyze DNA sequence. Nucleic Acids Res. 1999;27:573-80.

50. Nagaki K, Talbert PB, Zhong CX, Dawe RK, Henikoff S, Jiang J. Chromatin immunoprecipitation reveals that the 180 -bp satellite repeat is the key functional DNA element of Arabidopsis thaliana centromeres. Genetics. 2003;163:1221-5.

51. Edgar RC. MUSCLE: a multiple sequence alignment method with reduced time and space complexity. BMC Bioinformatics. 2004;5:113.

\section{Submit your next manuscript to BioMed Central and take full advantage of:}

- Convenient online submission

- Thorough peer review

- No space constraints or color figure charges

- Immediate publication on acceptance

- Inclusion in PubMed, CAS, Scopus and Google Scholar

- Research which is freely available for redistribution 